\title{
Frequency-Selective Predistortion Linearization of RF Power Amplifiers
}

\author{
Patrick Roblin, Member, IEEE, Suk Keun Myoung, Dominique Chaillot, Young Gi Kim, Member, IEEE, \\ Ayub Fathimulla, Jeff Strahler, and Steven Bibyk, Member, IEEE
}

\begin{abstract}
This paper presents a frequency-selective RF vector predistortion linearization system for RF multicarrier power amplifiers (PAs) affected by strong differential memory effects. Differential memory effects can be revealed in two-tone experiment by the divergence for increasing tone-spacing of the vector Volterra coefficients associated with the lower and upper intermodulations tones. Using large-signal vector measurement with a large-singal network analyzer, a class-AB LDMOS RF PA is demonstrated to exhibit a strong differential memory effect for modulation bandwidth above 0.3 MHz. New frequency-selective RF and baseband predistortion linearization algorithms are proposed to separately address the linearization requirements of the interband and inband intermodulation products of both the lower and upper sidebands. Theoretical verification of the algorithms are demonstrated with MATLAB simulations using a Volterra/Wiener PA model with memory effects. The baseband linearization algorithm is next implemented in a field-programmable gate array and experimentally investigated for the linearization of the class-AB LDMOS PA for two carrier wideband code-division multiple-access signals. The ability of the algorithm to selectively linearize the two interband and four inband intermodulation products is demonstrated. Adjacent channel leakage ratio of up to $45 \mathrm{dBc}$ for inband and interband are demonstrated experimentally at twice the typical fractional bandwidth.
\end{abstract}

Index Terms-Large-signal network analyzer (LSNA), linearization, memory effects, power amplifiers (PAs), predistortion, Volterra series.

\section{INTRODUCTION}

$\mathbf{O}$ NE OF the most challenging issues in designing RF power amplifiers (PAs) is the linearity requirement. The spurious emissions from nonlinear RF PAs are spread out over

Manuscript received May 11, 2007; revised September 24, 2007. This work was supported in part by the National Science Foundation (NSF) GOALI under Grant ECS-0622003 and by Epitaxial Technologies LLC under a grant.

P. Roblin and S. Bibyk are with the Department of Electrical and Computer Engineering, The Ohio State University, Columbus OH 43210 USA (e-mail: roblin@ece.osu.edu; bibyk@ece.osu.edu).

S. K. Myoung was with the Department of Electrical and Computer Engineering, The Ohio State University, Columbus OH 43210 USA. He is now with the System Core Technology Laboratory, Telecommunication Research and Development Center, Samsung, Suwon, 443-742 Gyeonggi, Korea (e-mail: myoungs@ee.eng.ohio-state.edu).

D. Chaillot is with the Department of Electrical and Computer Engineering, The Ohio State University, Columbus OH 43210 USA, on leave from the Commissariat à l'énergie atomique (CEA), 91191 Gif sur Yvette, France (e-mail: d.chaill@wanadoo.fr).

Y. G. Kim is with the Department of Electrical and Computer Engineering, The Ohio State University, Columbus OH 43210 USA, on leave from the Department of Data Communication Engineering, Anyang University, 430-714 Gyeonggi-do, Korea (e-mail: kimyo@ece.osu.edu; kimyg@ anyang.ac.kr).

A. Fathimulla is with Epitaxy Technologies LLC, Baltimore, MD 21227 USA (e-mail: afathimulla@epiwafers.com).

J. Strahler is with the Andrew Corporation, Westchester, IL 60154 USA (e-mail: jeff.strahler@andrew.com).

Color versions of one or more of the figures in this paper are available online at http://ieeexplore.ieee.org.

Digital Object Identifier 10.1109/TMTT.2007.912241 neighbor channels. As more complex modulation techniques such as wideband code division multiple access (WCDMA) or orthogonal frequency-division multiplexing (OFDM) and wider signal bandwidth are used, higher peak-to-average power ratios (PAPRs) (e.g., $4.5 \mathrm{~dB}$ for handset and $12 \mathrm{~dB}$ for basestation in WCDMA) result, imposing stronger linearity requirements on RF PAs. Memory effects also become significant in high-efficiency PAs operating with wideband signals and need to be taken into account for their linearization. Memory effects can be classified into two main types: slow memory effects and fast memory effects [1], [2]. Slow memory effects, which encompass temperature effects [3], traps, and aging are usually observed for bandwidth below $1 \mathrm{MHz}$, whereas fast memory effects, which typically originate from the intrinsic transistor, as well as matching and bias networks are usually observed above $1 \mathrm{MHz}$ [2].

The most performant broadband linearization systems have usually been based upon the feedforward technique [4]. However, RF and baseband predistortion linearization techniques have become an attractive solution owing to their reduced cost and complexity. For multicarrier PA applications, an effort has been placed to increase the bandwidth of predistortion linearization to combat fast memory effects. A direct approach relying on an RF predistortion algorithm with instantaneous adaptive feedback [5] has demonstrated improved performance. However, RF predistortion with instantaneous feedback remains limited by the computational speed of the digital signal processing (DSP) controller, and RF predistortion is affected, in general, by the timing mismatch between the input and predistortion signals of the vector modulator. On the other hand, model-based baseband predistortion algorithms, such as the frequency-selective algorithm presented here, do not require any such synchronization and can be designed to linearize RF PAs with fast memory effects while reserving narrowband adaptive feedback to slow memory effects.

The development of broadband predistorters is now facilitated by the availability of a variety of behavioral models of PAs (for a review, see [3] and [6]) accounting for memory effects. To address fast memory effects in PAs in a practical and efficient fashion, memory polynomials were introduced in baseband predistortion and demonstrated experimentally to improve the linearization bandwidth [7], [8]. A broadband baseband linearization implementation with memory polynomials combined with narrowband adaptive feedback was then demonstrated using a genetic adaptive algorithm [9].

In this paper, we present an alternative baseband predistortion approach, which divides the modulation bandwidth in several bands, effectively assuming that the frequency dependence of 
the PA nonlinearities is piecewise quasi-memoryless. Unlike a frequency-selective algorithm we previously reported [10], the new theoretically based model presented here is developed from a third Volterra PA model and has the capacity of differentiating between six types of inband and interband third-order intermodulation distortion (IMD3) processes instead of just the lower sideband (LSB) and upper sideband (USB).

This paper is organized as follows. First we will present in Section II the simple third-order Volterra PA model used for introducing differential memory effects. We will then experimentally characterize in Section III the differential memory effects taking place in the PA to be linearized. For this purpose, a large-signal network analyzer (LSNA) will be used to measure the power-dependent generalized third-order Volterra coefficients for a class-AB RF PA. Having demonstrated the large asymmetry between the LSB and USB generated by the nonlinearities, we will propose in Section IV a two-band frequency-selective RF predistortion algorithm, which can independently reduce four unwanted intermodulation bands generated by the PA for two-carrier WCDMA signals. In Section V, we will describe the baseband implementation of the frequency-selective predistortion linearization and present MATLAB simulation results for an amplifier with differential memory. In Section VI, we will present the field-programmable gate array (FPGA) test-bed and discuss the algorithms used for the experimental investigation. Using this digital test-bed, we will then experimentally demonstrate, in Section VII, the capability of the proposed frequency-selective vector predistortion (VPD) linearization to linearize the RF PA, which exhibits differential memory, as tested in Section III. Finally, in conclusion, we will summarize the results obtained and benchmark them.

\section{VOLTERRA/WIENER REPRESENTATION}

The most rigorous theory for including memory effects in not-strongly nonlinear systems is Volterra formalism [11]-[13]. In that formalism, the system is described by Volterra kernels of various orders. For example, a third-order kernel for a singleinput single-output (SISO) system is of the form

$$
\begin{array}{r}
y(t)=\iiint_{-\infty}^{\infty} h\left(\tau_{1}, \tau_{2}, \tau_{3}\right) x\left(t-\tau_{1}\right) x\left(t-\tau_{2}\right) \\
x\left(t-\tau_{3}\right) d \tau_{1} d \tau_{2} d \tau_{3} .
\end{array}
$$

A simpler picture emerges for Volterra series with periodic signals when we switch to the frequency domain. Let us assume initially the nonlinearities of the amplifier to be well represented by a third-order Volterra system. When a two-tone excitation $a_{1}\left(\omega_{1}\right)$ and $a_{1}\left(\omega_{2}\right)$ of frequency $\omega_{1}$ and $\omega_{2}$, respectively, is applied at port 1 , the output $b_{2}(\omega)$ at port 2 of the amplifier can be verified to be of the following form (see Fig. 1):

$$
\begin{aligned}
b_{2}\left(2 \omega_{1}-\omega_{2}\right)= & H_{3 m} a_{1}^{2}\left(\omega_{1}\right) a_{1}^{*}\left(\omega_{2}\right) \\
b_{2}\left(\omega_{1}\right)= & H_{1 m} a_{1}\left(\omega_{1}\right)+H_{3 m m} a_{1}\left(\omega_{1}\right)\left|a_{1}\left(\omega_{1}\right)\right|^{2} \\
& +H_{3 m p} a_{1}\left(\omega_{1}\right)\left|a_{1}\left(\omega_{2}\right)\right|^{2} \\
b_{2}\left(\omega_{2}\right)= & H_{1 p} a_{1}\left(\omega_{2}\right)+H_{3 p m} a_{1}\left(\omega_{2}\right)\left|a_{1}\left(\omega_{1}\right)\right|^{2} \\
& +H_{3 p p} a_{1}\left(\omega_{2}\right)\left|a_{1}\left(\omega_{2}\right)\right|^{2} \\
b_{2}\left(2 \omega_{2}-\omega_{1}\right)= & H_{3 p} a_{1}^{2}\left(\omega_{2}\right) a_{1}^{*}\left(\omega_{1}\right) .
\end{aligned}
$$

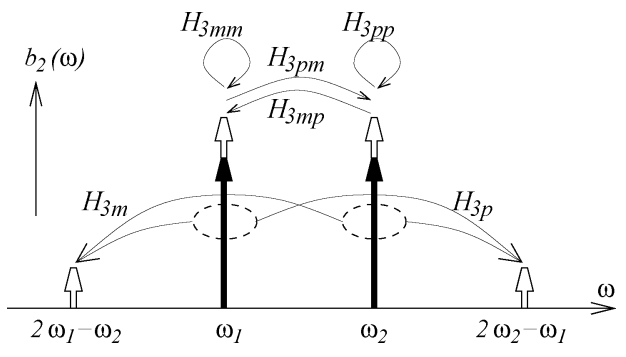

Fig. 1. Third-order intermodulation for two-tone excitation.

The output features the two desired tones plus two intermodulation tones at $2 \omega_{1}-\omega_{2}$ and $2 \omega_{2}-\omega_{1}$. The complex coefficients $H_{1 m}, H_{1 p}, H_{3 m}, H_{3 p}, H_{3 m m}, H_{3 m p}, H_{3 p m}$, and $H_{3 p p}$, which are calculated from the third Volterra series can be measured using an LSNA. For a memoryless PA with $y(t)=$ $T_{1} x(t)+T_{3} x^{3}(t)$, we have $H_{1 m}=H_{1 p}=T_{1}, H_{3 m}=H_{3 p}=$ $H_{3 m m}=H_{3 p p}=3 T_{3}$, and $H_{3 m p}=H_{3 p m}=6 T_{3}$.

The Volterra series has been extended to larger input power for stochastic input signals with constant average power levels by Wiener [12]. For larger deterministic input signals, one can also generalize the Volterra formalism by making the $H$ coefficients introduced above power dependent.

\section{NONLINEAR PA CHARACTERIZATION WITH LSNA}

The nonlinear characterization of the PA can be performed using an LSNA, as shown in Figs. 2-4. With an LSNA, we can measure the amplitude and phase of the incident and the transmitted periodically modulated waves at the fundamental and harmonics [14]. In this measurement, the vector source generator (ESG 4438C) is synchronized with the LSNA 10-MHz reference clock.

In this study, the LSNA is used to characterize the third-order intermodulation response of a class-AB LDMOSFET 10-W PA at $895 \mathrm{MHz}$ for two-tone excitation [10]. The data acquisition is performed under Labwindows control. The LSNA is used to measure both the amplitude and phase of the two-tone RF excitation $a_{1}\left(\omega_{1}\right)$ and $a_{1}\left(\omega_{2}\right)$ incident on port 1 , as well as the amplitude and phase of the transmitted intermodulation RF signals $b_{2}\left(2 \omega_{1}-\omega_{2}\right), b_{2}\left(\omega_{1}\right), b_{2}\left(\omega_{2}\right)$, and $b_{2}\left(2 \omega_{2}-\omega_{1}\right)$ transmitted to port 2. Using these intermodulation signals, we calculated next the generalized Volterra/Wiener coefficients $H_{3 m}$ and $H_{3 p}$ defined in (5) and (6) as follows:

$$
\begin{aligned}
H_{3 m}\left(\omega_{m},\left|a_{1}\right|\right) & =\frac{b_{2}\left(2 \omega_{1}-\omega_{2}\right)}{a^{2}{ }_{1}\left(\omega_{1}\right) a^{*}{ }_{1}\left(\omega_{2}\right)} \\
H_{3 p}\left(\omega_{m},\left|a_{1}\right|\right) & =\frac{b_{2}\left(2 \omega_{2}-\omega_{1}\right)}{a^{*}{ }_{1}\left(\omega_{1}\right) a^{2}{ }_{1}\left(\omega_{2}\right)}
\end{aligned}
$$

with $\omega_{m}=\omega_{2}-\omega_{1}$ and assuming $\left|a_{1}\left(\omega_{1}\right)\right|=\left|a_{1}\left(\omega_{2}\right)\right|=\left|a_{1}\right|$. Note that these coefficients are found to be reproducible measurement after measurement [10]. In the limit of small $\left|a_{1}\right|$, these coefficients reduce to the usual power-independent Volterra coefficients.

The amplitude and phase of these coefficients are plotted in Fig. 2, respectively, as a function of tone spacing $\omega_{m}$ and input power $\left|a_{1}\left(\omega_{1}\right)\right|^{2}=\left|a_{1}\left(\omega_{2}\right)\right|^{2}$ from -4 to $6 \mathrm{dBm}$. As shown in Fig. 2, the variation of $H_{3 m}$ and $H_{3 p}$ as a function 

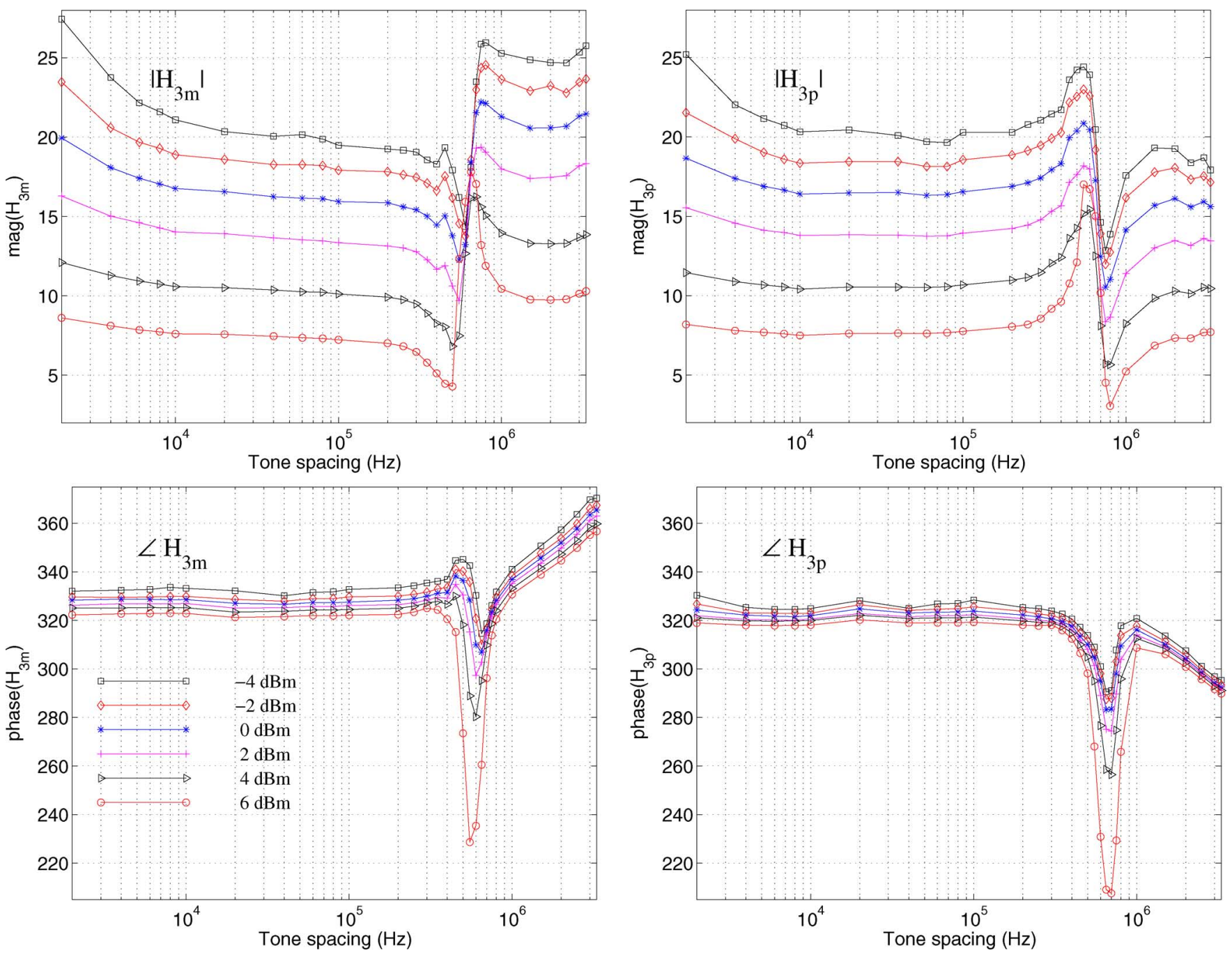

Fig. 2. Comparison of amplitude and phase of $H_{3 m}$ and $H_{3 p}$ versus the tone spacing $\omega_{m}$ for different power levels (-4-6 dBm) (data from [10]).
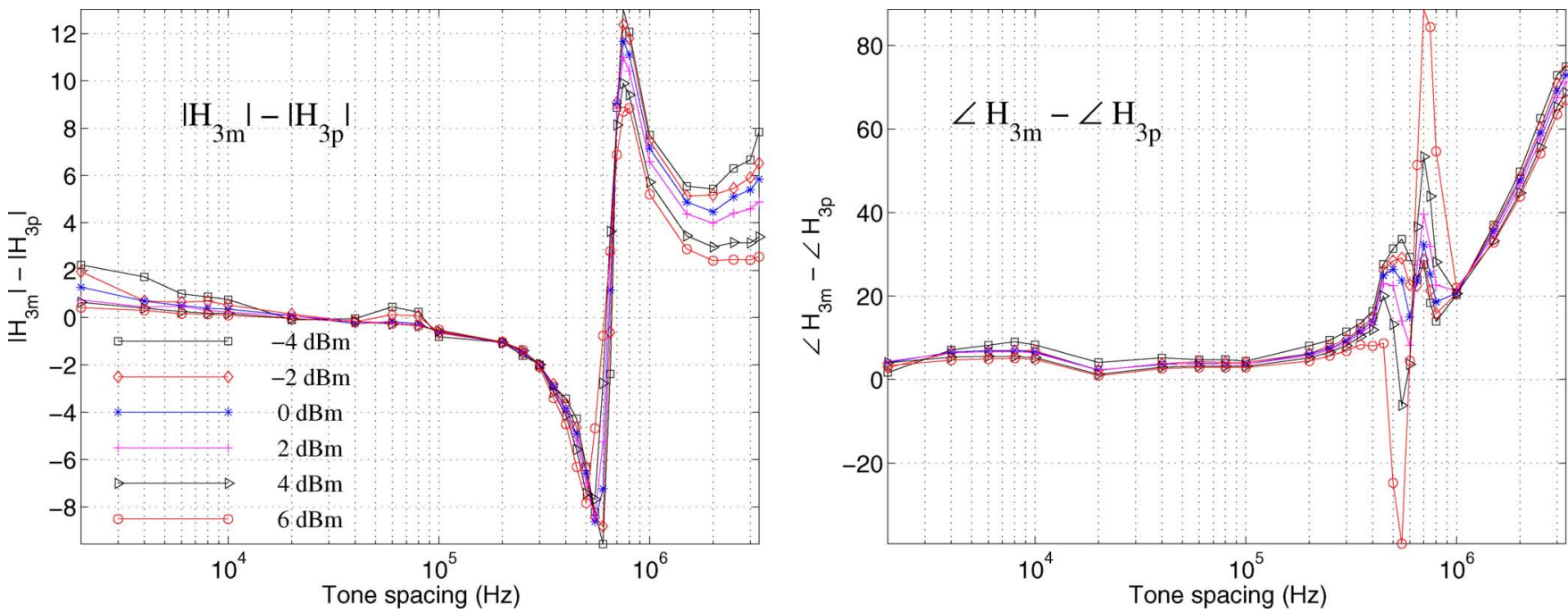

Fig. 3. Difference between $H_{3 m}$ and $H_{3 p}$ versus $\omega_{m}=\omega_{2}-\omega_{1}$ reveals a strong differential memory effect above $1 \mathrm{MHz}$ (data from [10]).

of tone spacing $\omega_{m}$ reveals the presence of memory effects (frequency dependent nonlinearity) in the PA. Above $0.3 \mathrm{MHz}$, the differences in amplitude and phase plotted in Fig. 3 increase rapidly with tone spacing. This is to be referred to as differential memory. These generalized Volterra/Wiener coefficients indicate that memoryless linearization techniques will not perform 


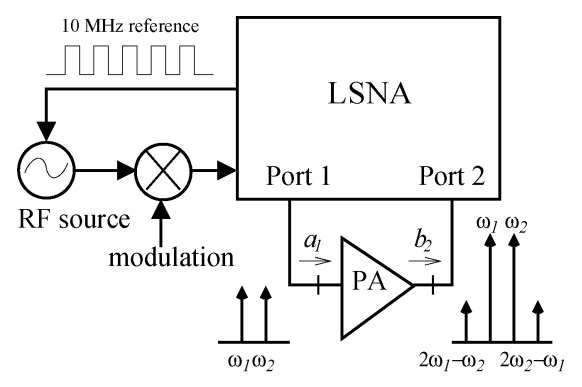

Fig. 4. Large-signal test-bed used for nonlinear characterization.

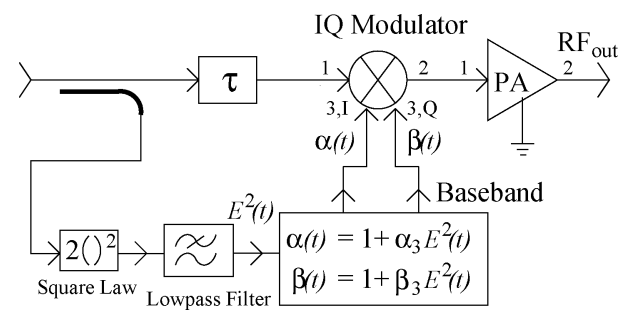

Fig. 5. Scalar RF predistortion.

optimally for this PA for bandwidths above $0.3 \mathrm{MHz}$ [10]. Note that the power dependence originates from the contribution of higher order nonlinearities (fifth, seventh, etc. orders) in the PA. The applicability of this generalized Volterra/Wiener model, which is adopted for the remainder of this paper, will be further validated for the PA considered by the ultimate linearization achieved.

\section{RF VPD}

A conventional predistortion linearization system with thirdorder scalar compensation is shown in Fig. 5.

The ideal in-phase and quadrature (IQ) modulator used in this predistortion system is shown in Fig. 6. The waveforms $\alpha(t)$ and $\beta(t)$ calculated from the signal envelope $E^{2}(t)$ are injected at the I and Q baseband inputs of the IQ modulator. Consider a two-tone excitation $x(t)=x_{L}(t)+x_{U}(t)$ at port 1 of the modulator

$$
x(t)=2 \operatorname{Re}\left[a_{1}\left(\omega_{1}\right) \exp \left(j \omega_{1} t\right)+a_{1}\left(\omega_{2}\right) \exp \left(j \omega_{2} t\right)\right]
$$

where $a_{1}(\omega)$ are the Fourier coefficient weights

$$
\begin{aligned}
X(\omega)=\mathcal{F}[x(t)]=a_{1} & \left(\omega_{1}\right) \delta\left(f-f_{1}\right)+a_{1}\left(\omega_{2}\right) \delta\left(f-f_{2}\right) \\
& +a_{1}^{*}\left(\omega_{1}\right) \delta\left(f+f_{1}\right)+a_{1}^{*}\left(\omega_{2}\right) \delta\left(f+f_{2}\right) .
\end{aligned}
$$

The envelope $E^{2}(t)$ obtained by low-pass filtering $2 x^{2}(t)$ can be expanded in terms of inband $E_{\text {inband }}^{2}$ and interband $E_{\text {inter }}^{2}$ components

$$
\begin{aligned}
E^{2}(t) & =E_{\text {inband }}^{2}+E_{\text {inter }}^{2}(t) \\
E_{\text {inband }}^{2} & =4\left|a_{1}\left(\omega_{1}\right)\right|^{2}+4\left|a_{1}\left(\omega_{2}\right)\right|^{2} \\
E_{\text {inter }}^{2}(t) & =2 \operatorname{Re}\left[a_{E}\left(\omega_{m}\right) \exp \left(j \omega_{m} t\right)\right] \\
a_{E}\left(\omega_{m}\right) & =4 a_{1}\left(\omega_{2}\right) a_{1}^{*}\left(\omega_{1}\right)
\end{aligned}
$$

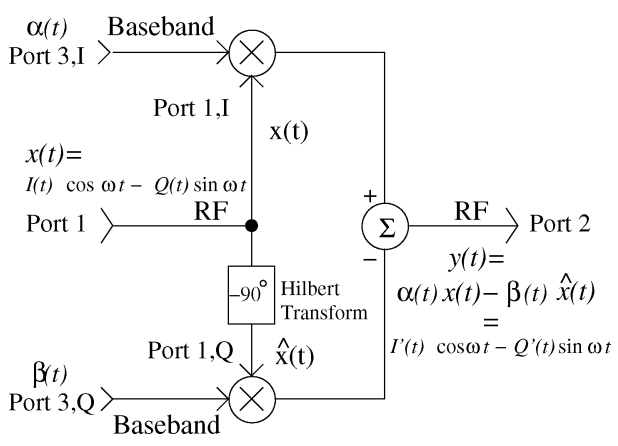

Fig. 6. Ideal IQ modulator used in RF predistortion.

with $\omega_{m}=\omega_{2}-\omega_{1}$. The RF scalar predistortion (SPD) topology shown in Fig. 5 is not capable of addressing independently the inband and interband distortions. Note that using higher order terms $\alpha_{5} E^{4}(t)$ and $\beta_{5} E^{4}(t)$ in $\alpha(t)$ and $\beta(t)$, respectively, can only bring a valid third-order correction at a single power level at a time. To allow for the additional degrees of freedom required for piecewise quasi-memoryless PAs, we shall introduce the RF VPD topology shown in Fig. 7.

Let us first consider the interband linearization block, which relies solely on the interband envelope $E_{\text {inter }}^{2}$. For added generality, we allow $\alpha_{3}$ and $\beta_{3}$ to be complex coefficients (phase shifting of $\left.E_{\text {inter }}^{2}\right)$. The excitations $\alpha_{\text {inter }}(t)$ and $\beta_{\text {inter }}(t)$ are then generalized to

$$
\begin{aligned}
\alpha_{\text {inter }}(t) & =\alpha_{0}+2 \operatorname{Re}\left[\begin{array}{ll}
\alpha_{3} & a_{E}\left(\omega_{m}\right) \exp \left(j \omega_{m} t\right)
\end{array}\right] \\
\beta_{\text {inter }}(t) & =\beta_{0}+2 \operatorname{Re}\left[\begin{array}{ll}
\beta_{3} & a_{E}\left(\omega_{m}\right) \exp \left(j \omega_{m} t\right)
\end{array}\right]
\end{aligned}
$$

with $\alpha_{0}=\beta_{0}=1$ and $a_{E}\left(\omega_{m}\right)$, the phasor associated with the interband envelope $E_{\text {inter }}^{2}(t)$ of the two-tone excitation. The output of the modulator is then

$$
y_{\text {inter }}(t)=\alpha_{\text {inter }}(t) x(t)-\beta_{\text {inter }}(t) \widehat{x(t)}
$$

where $\widehat{x(t)}=\mathcal{H}[x(t)]$ denotes the Hilbert of $x(t)$.

The inband signal at the output of the IQ modulator is then

$$
y_{\text {inter }}\left(\omega_{1}\right)=\left(\alpha_{0}+j \beta_{0}\right) a_{1}\left(\omega_{1}\right)+\left(\alpha_{3}^{*}+j \beta_{3}^{*}\right) 4 a_{1}\left(\omega_{1}\right)\left|a_{1}\left(\omega_{2}\right)\right|^{2}
$$

$y_{\text {inter }}\left(\omega_{2}\right)=\left(\alpha_{0}+j \beta_{0}\right) a_{1}\left(\omega_{2}\right)+\left(\alpha_{3}+j \beta_{3}\right) 4 a_{1}\left(\omega_{2}\right)\left|a_{1}\left(\omega_{1}\right)\right|^{2}$.

Similarly, the IMD3 at the output of the IQ modulator is

$$
\begin{aligned}
& y_{\text {inter }}\left(2 \omega_{1}-\omega_{2}\right)=\left[\alpha_{3}^{*}+j \beta_{3}^{*}\right] 4 a_{1}^{2}\left(\omega_{1}\right) a_{1}^{*}\left(\omega_{2}\right) \\
& y_{\text {inter }}\left(2 \omega_{2}-\omega_{1}\right)=\left[\alpha_{3}+j \beta_{3}\right] 4 a_{1}^{2}\left(\omega_{2}\right) a_{1}^{*}\left(\omega_{1}\right) .
\end{aligned}
$$

Next the IMD3 correction signals $y_{\text {inter }}\left(2 \omega_{1}-\omega_{2}\right)$ and $y_{\text {inter }}\left(2 \omega_{2}-\omega_{1}\right)$ generated by the IQ modulator and amplified by the amplifier are superposed at the output of the PA with the IMD3 terms generated by the PA so that they cancel as follows:

$$
\begin{aligned}
b_{2, \mathrm{PA}}\left(2 \omega_{1}-\omega_{2}\right)= & H_{3 m} y_{\text {inter }}^{2}\left(\omega_{1}\right) y_{\text {inter }}^{*}\left(\omega_{2}\right) \\
& +H_{1 m} y_{\text {inter }}\left(2 \omega_{1}-\omega_{2}\right) \\
= & 0
\end{aligned}
$$




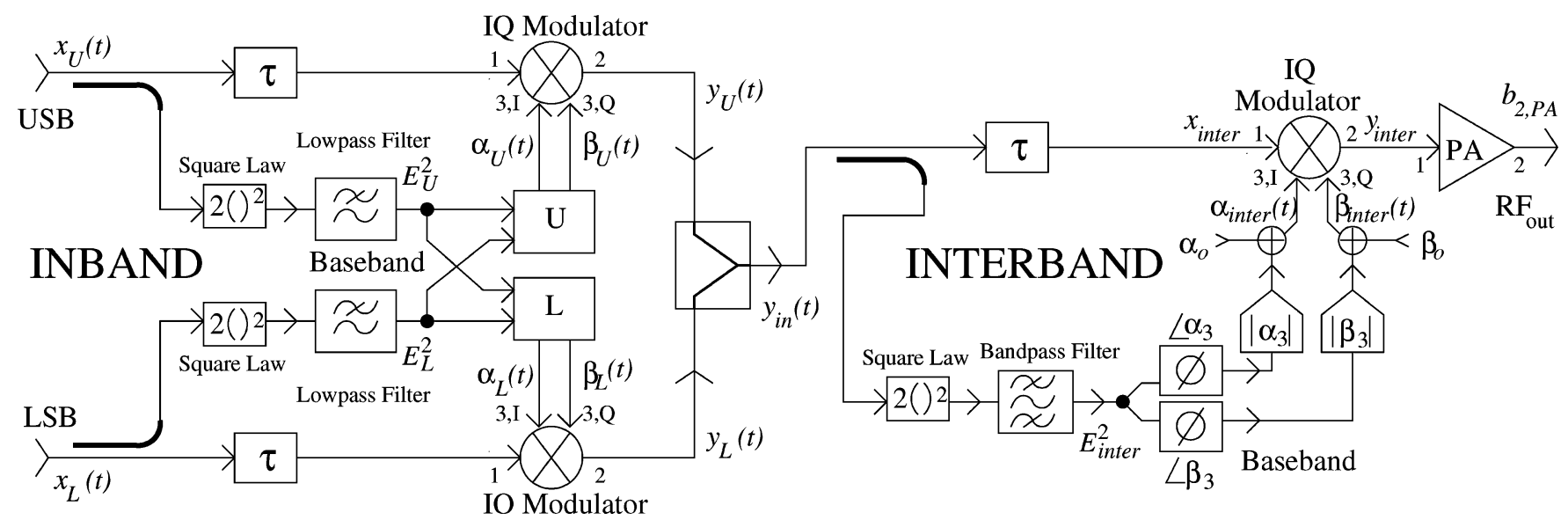

Fig. 7. RF VPD implementation for two bands featuring the LSB and USB inband stages followed by the interband stage.

$$
\begin{aligned}
b_{2, \mathrm{PA}}\left(2 \omega_{2}-\omega_{1}\right)= & H_{3 p} y_{\text {inter }}^{*}\left(\omega_{1}\right) y_{\text {inter }}^{2}\left(\omega_{2}\right) \\
& +H_{1 p} \quad y_{\text {inter }}\left(2 \omega_{2}-\omega_{1}\right) \\
= & 0 .
\end{aligned}
$$

Retaining only the leading terms in (8) and (9), the following products can be evaluated:

$$
\begin{aligned}
y_{\text {inter }}^{2}\left(\omega_{1}\right) y_{\text {inter }}^{*}\left(\omega_{2}\right) & \simeq(1+j)^{2}(1-j) a_{1}^{2}\left(\omega_{1}\right) a_{1}^{*}\left(\omega_{2}\right) \\
& \simeq 2(1+j) a_{1}^{2}\left(\omega_{1}\right) a_{1}^{*}\left(\omega_{2}\right) \\
y_{\text {inter }}^{*}\left(\omega_{1}\right) y_{\text {inter }}^{2}\left(\omega_{2}\right) & =(1+j)^{2}(1-j) a_{1}^{2}\left(\omega_{2}\right) a_{1}^{*}\left(\omega_{1}\right) \\
& =2(1+j) a_{1}^{2}\left(\omega_{2}\right) a_{1}^{*}\left(\omega_{1}\right) .
\end{aligned}
$$

Substituting (14), (15), (10), and (11) into (12) and (13) we obtain the following systems of equations:

$$
\begin{aligned}
& \alpha_{3}-j \beta_{3}=-\frac{1}{2}(1-j) \frac{H_{3 m}^{*}}{H_{1 m}^{*}}=Z_{1} \\
& \alpha_{3}+j \beta_{3}=-\frac{1}{2}(1+j) \frac{H_{3 p}}{H_{1 p}}=Z_{2} .
\end{aligned}
$$

This linear system is easily solved for $\alpha_{3}$ and $\beta_{3}$ as follows:

$$
\alpha_{3}=\frac{1}{2}\left(Z_{1}+Z_{2}\right) \text { and } \beta_{3}=\frac{1}{2 j}\left(Z_{2}-Z_{1}\right) .
$$

In general, the coefficients $\alpha_{3}$ and $\beta_{3}$ are complex numbers. This implies that the baseband envelope signal sent to the port 3I and $3 \mathrm{Q}$ of the IQ modulator must not only be scaled, but also phase shifted. This is referred in this paper as VPD linearization.

For a quasi-memoryless PA, the following identity holds:

$$
\begin{aligned}
& H_{3 m}=H_{3 p}=H_{3} \\
& H_{1 m}=H_{1 p}=H_{1} .
\end{aligned}
$$

It results that we have $Z_{1}=Z_{2}^{*}=Z$, and the $\alpha_{3}$ and $\beta_{3}$ coefficients are simply given for quasi-memoryless PA by

$$
\begin{aligned}
& \alpha_{3}=-\frac{1}{2} \operatorname{Re}\left[(1+j) \frac{H_{3}}{H_{1}}\right] \\
& \beta_{3}=-\frac{1}{2} \operatorname{Im}\left[(1+j) \frac{H_{3}}{H_{1}}\right] .
\end{aligned}
$$

Note that $\alpha_{3}$ and $\beta_{3}$ are now both real. No phase shift is required and the conventional SPD linearization is sufficient for such quasi-memoryless PAs.

The above linearization applies only to the cancellation of the interband terms of (1) and (4) generated by the PA, which corrupt adjacent bands. Let us consider now the cancellation of the inband terms (2) and (3) generated by the PA, which degrades the error vector measurement (EVM).

First let us define the $\alpha_{L}, \beta_{L}, \alpha_{U}$, and $\beta_{U}$ control signals on the inband modulators

$$
\begin{aligned}
& \alpha_{L}=1+\alpha_{L L} E_{L}^{2}+\alpha_{L U} E_{U}^{2} \\
& \beta_{L}=0+\beta_{L L} E_{L}^{2}+\beta_{L U} E_{U}^{2} \\
& \alpha_{U}=1+\alpha_{U L} E_{L}^{2}+\alpha_{U U} E_{U}^{2} \\
& \beta_{U}=0+\beta_{U L} E_{L}^{2}+\beta_{U U} E_{U}^{2}
\end{aligned}
$$

where we introduced $E_{U}^{2}=4\left|a_{1}\left(\omega_{2}\right)\right|^{2}$ and $E_{L}^{2}=4\left|a_{1}\left(\omega_{1}\right)\right|^{2}$, the LSB and USB components of the inband envelope $E_{\text {inband }}^{2}=E_{L}^{2}+E_{U}^{2}$. The outputs of the inband modulators for the two-tone input $x(t)=x_{L}(t)+x_{U}(t)$ are

$$
\begin{aligned}
& y_{L}(t)=\alpha_{L}(t) x_{L}(t)-\beta_{L}(t) \widehat{x_{L}(t)} \\
& y_{U}(t)=\alpha_{U}(t) x_{U}(t)-\beta_{U}(t) \widehat{x_{U}(t)} .
\end{aligned}
$$

We now need to calculate the required $\alpha_{L / U, L / U}$ and $\beta_{L / U, L / U}$ control coefficients. We start from the output signal of the PA at port 2 as follows:

$$
\begin{aligned}
b_{2, \mathrm{PA}}\left(\omega_{1}\right)= & H_{1 m} y_{\text {inter }}\left(\omega_{1}\right) \\
& +H_{3 m m} y_{\text {inter }}\left(\omega_{1}\right)\left|y_{\text {inter }}\left(\omega_{1}\right)\right|^{2} \\
& +H_{3 m p} y_{\text {inter }}\left(\omega_{1}\right)\left|y_{\text {inter }}\left(\omega_{2}\right)\right|^{2} \\
b_{2, \mathrm{PA}}\left(\omega_{2}\right)= & H_{1 p} y_{\text {inter }}\left(\omega_{2}\right) \\
& +H_{3 p m} y_{\text {inter }}\left(\omega_{2}\right)\left|y_{\text {inter }}\left(\omega_{1}\right)\right|^{2} \\
& +H_{3 p p} y_{\text {inter }}\left(\omega_{2}\right)\left|y_{\text {inter }}\left(\omega_{2}\right)\right|^{2} .
\end{aligned}
$$


In the above equations, $y_{\text {inter }}(t)$, the PA input, is the output signal from the interband modulator, which is expressed in the frequency domain using (8) and (9) as

$$
\begin{aligned}
y_{\text {inter }}\left(\omega_{1}\right)= & (1+j) y_{L}\left(\omega_{1}\right) \\
& +4\left(\alpha_{3}^{*}+j \beta_{3}^{*}\right) y_{L}\left(\omega_{1}\right)\left|y_{U}\left(\omega_{2}\right)\right|^{2} \\
y_{\text {inter }}\left(\omega_{2}\right)= & (1+j) y_{U}\left(\omega_{2}\right) \\
& +4\left(\alpha_{3}+j \beta_{3}\right) y_{U}\left(\omega_{2}\right)\left|y_{L}\left(\omega_{1}\right)\right|^{2} .
\end{aligned}
$$

Note that care was taken to include the parasitic inband component introduced by the interband modulator. The $y_{L}(t)$ and $y_{U}(t)$ signals are themselves the output of the inband modulator $L$ and $U$ expressed in the frequency domain by

$$
\begin{aligned}
& y_{L}\left(\omega_{1}\right)=a_{1}\left(\omega_{1}\right) \times\left(\alpha_{L}+j \beta_{L}\right) \\
& y_{U}\left(\omega_{2}\right)=a_{1}\left(\omega_{2}\right) \times\left(\alpha_{U}+j \beta_{U}\right) .
\end{aligned}
$$

We can now evaluate the control coefficients required for the cancellation of the leading inband distortion generated by the PA and the interband modulator to yield the PA output $b_{2, \mathrm{PA}}\left(\omega_{1}\right) \simeq$ $(1+j) a_{1}\left(\omega_{1}\right)$ and $b_{2, \mathrm{PA}}\left(\omega_{2}\right) \simeq(1+j) a_{1}\left(\omega_{2}\right)$

$$
\begin{aligned}
& \gamma_{L L}=\alpha_{L L}+j \beta_{L L}=-\frac{H_{3 m m}}{2 H_{1 m}} \\
& \gamma_{L U}=\alpha_{L U}+j \beta_{L U}=-\frac{H_{3 m p}}{2 H_{1 m}}-\frac{\alpha_{3}^{*}+j \beta_{3}^{*}}{1+j} \\
& \gamma_{U L}=\alpha_{U L}+j \beta_{U L}=-\frac{H_{3 p m}}{2 H_{1 p}}-\frac{\alpha_{3}+j \beta_{3}}{1+j} \\
& \gamma_{U U}=\alpha_{U U}+j \beta_{U U}=-\frac{H_{3 p p}}{2 H_{1 p}} .
\end{aligned}
$$

It is to be noted that the inband linearization is affected by the interband linearization and it is necessary to first obtain the interband linearization coefficients $\alpha_{3}$ and $\beta_{3}$ to implement the final inband linearization.

\section{BASEBAND VPD}

The derivation given above was conducted for simplicity assuming a two-tone excitation. In practice, the RF carrier is digitally modulated. The two-tone analysis is applicable to a twocarrier system where $\omega_{1}$ and $\omega_{2}$ are the center frequency. Consider the simplest case where each of the modulated carriers can be represented with two tones per band, as shown in Fig. 8. Four tones (plain lines) are, therefore, injected at the input of the PA and 16 tones are observed at the output. This four-tone excitation permits us to clearly distinguish the interband and inband intermodulation distortions generated by the various $H_{3 m}, H_{3 p}$, $H_{3 m m}, H_{3 m p}, H_{3 p m}$, and $H_{3 p p}$ coefficients. In the previous derivation, we focused on an RF implementation of VPD. Here, we will present a baseband implementation. For this purpose, we shall recast the VPD theory in terms of the $I(t)$ and $Q(t)$ signal representation.

Baseband predistortion is implemented by directly predistorting the $I$ and $Q$ signals. For example, in the baseband implementation, the analog IQ modulator in Fig. 6,

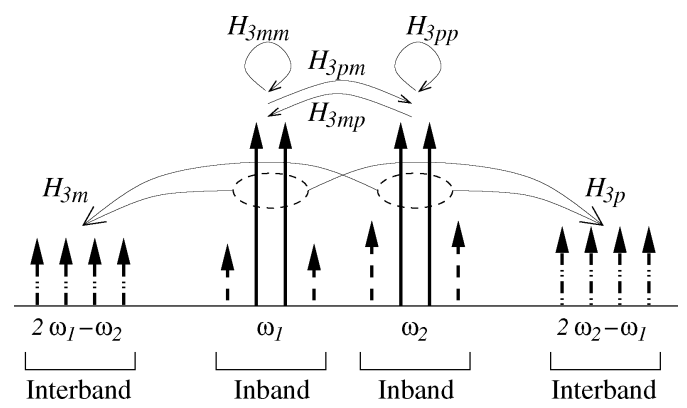

Fig. 8. Inband and interband third-order intermodulation in two-band model.

is effectively replaced by a matrix multiplication of the form

$$
\left[\begin{array}{c}
I^{\prime}(t) \\
Q^{\prime}(t)
\end{array}\right]=\left[\begin{array}{cc}
\alpha(t) & -\beta(t) \\
\beta(t) & \alpha(t)
\end{array}\right]\left[\begin{array}{c}
I(t) \\
Q(t)
\end{array}\right]
$$

using

$$
\begin{aligned}
& x(t)=I(t) \cos (\omega t)-Q(t) \sin (\omega t) \\
& y(t)=I^{\prime}(t) \cos (\omega t)-Q^{\prime}(t) \sin (\omega t) .
\end{aligned}
$$

For example, for the two-tone excitation of (7), we have

$$
I(t)=2 \operatorname{Re}[z(t)] \text { and } Q(t)=2 \operatorname{Im}[z(t)]
$$

with

$$
z(t)=a_{1}\left(\omega_{1}\right) \exp \left(-j \frac{\omega_{m}}{2} t\right)+a_{1}\left(\omega_{2}\right) \exp \left(j \frac{\omega_{m}}{2} t\right) .
$$

In the RF VPD, we introduced a dc block in the calculation of the interband envelope $E_{\text {inter }}$. The dc components, which contains important data, were used in the inband intermodulation linearization. This frequency-domain manipulation is one of the requirements for the implementation of frequency-selective predistortion accounting for differential memory effect. Alternatively, in the baseband implementation, we will effectively separate the incoming frequency spectrum in terms of the LSB $\left(f<f_{c}\right)$ and the USB $\left(f>f_{c}\right)$ with $f_{c}$ being the carrier.

To perform this two-band filtering, the easiest is to partition the input $I$ and $Q$ in terms of their USB and LSB components as follows:

$$
\begin{aligned}
I(t) & =I_{U}(t)+I_{L}(t) \\
Q(t) & =Q_{U}(t)+Q_{L}(t) \\
Q_{U} & =\widehat{I_{U}} \\
Q_{L} & =-\widehat{I_{L}} .
\end{aligned}
$$

Note that we use the notation $\widehat{I(t)}=\mathcal{H}[I(t)]$ to denote the Hilbert of $I(t)$. One can readily verify that $I_{U}, I_{L}, \widehat{I_{U}}$, and $\widehat{I_{L}}$ are obtained from $I$ and $Q$ and their Hilbert using

$$
I_{L}=\frac{I+\widehat{Q}}{2} \quad \widehat{I_{L}}=\frac{\widehat{I}-Q}{2} \quad I_{U}=\frac{I-\widehat{Q}}{2} \quad \widehat{I_{U}}=\frac{\widehat{I}+Q}{2} .
$$

The pair $I_{U}$ and $Q_{U}$ generates the USB $\left(f>f_{c}\right)$ and the pair $I_{L}$ and $Q_{L}$ generates the LSB $\left(f<f_{c}\right)$. We can evaluate the envelope in terms of this two-band IQ representation

$$
E^{2}(t)=I^{2}(t)+Q^{2}(t)=E_{\text {inter }}^{2}(t)+E_{\text {inband }}^{2}(t)
$$

$$
y(t)=\alpha(t) x(t)-\beta(t) \widehat{x(t)}
$$




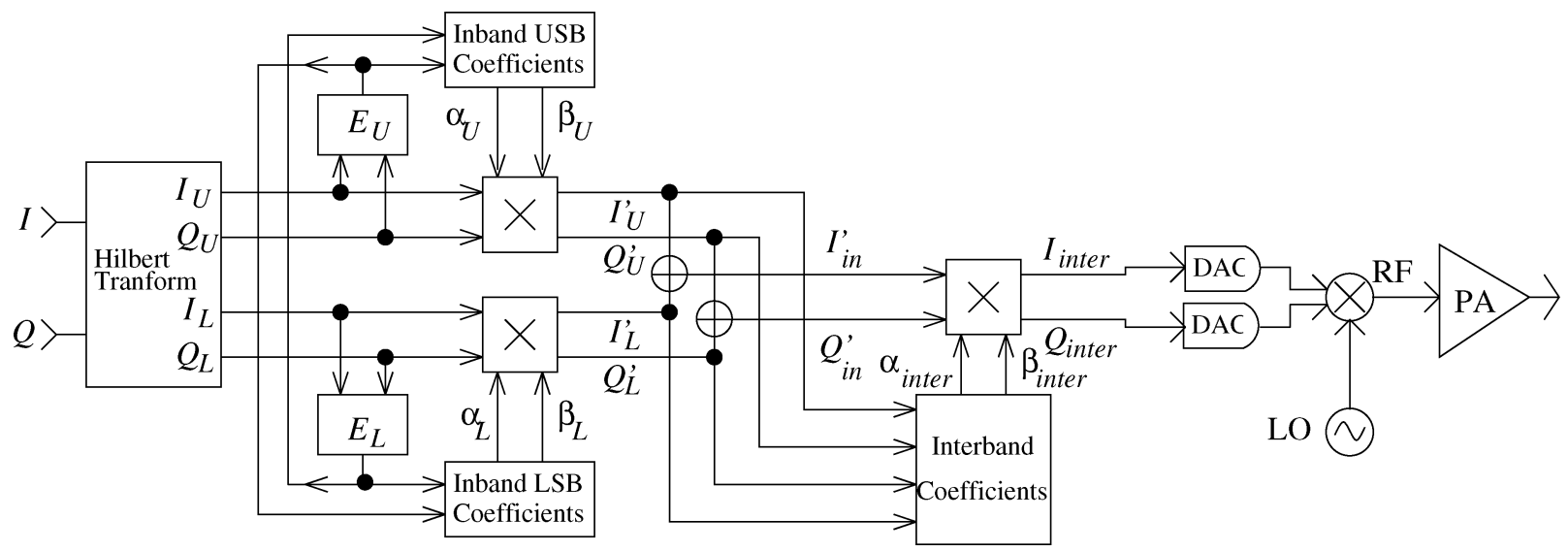

Fig. 9. Baseband VPD implementation for two bands featuring the LSB and USB inband stages followed by the interband stage.

where $E_{\text {inter }}^{2}$ and $E_{\text {inband }}^{2}$ are interband and inband envelope components defined as

$$
\begin{aligned}
E_{\text {inter }}^{2}(t) & =2 I_{U}(t) I_{L}(t)-2 \widehat{I_{U}(t)} \widehat{I_{L}(t)} \\
E_{\text {inband }}^{2}(t) & =E_{U}^{2}(t)+E_{L}^{2}(t) \\
E_{U}^{2}(t) & =I_{U}^{2}(t)+{\widehat{I_{U}(t)}}^{2} \\
E_{L}^{2}(t) & \left.=I_{L}^{2}(t)+\widehat{I_{L}(t)}\right)^{2} .
\end{aligned}
$$

For a two-tone excitation, one can verify that $E_{\text {inband }}^{2}$ and $E_{\text {inter }}^{2}$ are the dc and ac components, respectively, of the envelope. When dealing with general multitone signals, they define the time-dependent envelopes needed, respectively, to correct for the inband and interband intermodulation distortions, as shown in Fig. 8.

The $E_{\text {inband }}^{2}$ terms are used to first linearize the LSB $I_{L}$ and $Q_{L}$ and the USB $I_{U}$ and $Q_{U}$ using the $\alpha_{L}(t), \beta_{L}(t), \alpha_{U}(t)$, and $\beta_{U}(t)$ coefficients

$$
\begin{aligned}
\alpha_{L}(t) & =1+\operatorname{Re}\left[\gamma_{L L}\right] E_{L}^{2}(t)+\operatorname{Re}\left[\gamma_{L U}\right] E_{U}^{2}(t) \\
\beta_{L}(t) & =0+\operatorname{Im}\left[\gamma_{L L}\right] E_{L}^{2}(t)+\operatorname{Im}\left[\gamma_{L U}\right] E_{U}^{2}(t) \\
\alpha_{U}(t) & =1+\operatorname{Re}\left[\gamma_{U L}\right] E_{L}^{2}(t)+\operatorname{Re}\left[\gamma_{U U}\right] E_{U}^{2}(t) \\
\beta_{U}(t) & =0+\operatorname{Im}\left[\gamma_{U L}\right] E_{L}^{2}(t)+\operatorname{Im}\left[\gamma_{U U}\right] E_{U}^{2}(t) .
\end{aligned}
$$

The resulting $I_{U}^{\prime}, Q_{U}^{\prime}, I_{L}^{\prime}$, and $Q_{L}^{\prime}$ after the digital IQ modulator are

$$
\begin{aligned}
I_{L}^{\prime} & =\alpha_{L} I_{L}-\beta_{L} Q_{L} \\
Q_{L}^{\prime} & =\beta_{L} I_{L}+\alpha_{L} Q_{L}=-\widehat{I_{L}^{\prime}} \\
I_{U}^{\prime} & =\alpha_{U} I_{U}-\beta_{U} Q_{U} \\
Q_{U}^{\prime} & =\beta_{U} I_{U}+\alpha_{U} Q_{U}=\widehat{I_{U}^{\prime}} .
\end{aligned}
$$

The output of the inband predistortion linearization is then obtained by reconstituting $I$ and $Q$

$$
\begin{gathered}
I_{\text {in }}^{\prime}=I_{U}^{\prime}+I_{L}^{\prime} \\
Q_{\text {in }}^{\prime}=Q_{U}^{\prime}+Q_{L}^{\prime} .
\end{gathered}
$$

This inband algorithm is schematically represented in Fig. 9.

Next we need to calculate the interband intermodulation correction. In the Volterra theory, this correction is added to the inband intermodulation correction we have just calculated. It can be verified both experimentally and in simulations that it is preferable to proceed with the interband intermodulation correction using the interband envelope $E^{\prime 2}$ inter calculated using $I_{\text {in }}^{\prime}$ and $Q_{\text {in }}^{\prime}$ instead of $I$ and $Q$ since they have already been updated by the inband correction.

The interband digital modulator is fed the cross product terms $E^{\prime 2}$ inter of the signal envelope

$$
\begin{aligned}
& \alpha_{\text {inter }}(t)=1+\operatorname{Re}\left(\alpha_{3}\right) E^{\prime 2} \text { inter }-\operatorname{Im}\left(\alpha_{3}\right) \widehat{E^{\prime 2} \text { inter }} \\
& \beta_{\text {inter }}(t)=1+\operatorname{Re}\left(\beta_{3}\right) E^{\prime 2} \text { inter }-\operatorname{Im}\left(\beta_{3}\right) \widehat{E^{\prime 2} \text { inter }}
\end{aligned}
$$

where, according to the above discussion, we define

$$
E^{\prime 2}{ }_{\text {inter }}(t)=2 I_{U}^{\prime} I_{L}^{\prime}-2 \widehat{I_{U}^{\prime}} \widehat{I_{L}^{\prime}}
$$

The output IQ is then obtained from the digital modulator

$$
\begin{aligned}
I_{\text {inter }}^{\prime} & =\alpha_{\text {inter }} I_{\text {in }}^{\prime}-\beta_{\text {inter }} Q_{\text {in }}^{\prime} \\
Q_{\text {inter }}^{\prime} & =\alpha_{\text {inter }} Q_{\text {in }}^{\prime}+\beta_{\text {inter }} I_{\text {in }}^{\prime}
\end{aligned}
$$

The calculation of $\alpha_{\text {inter }}$ and $\beta_{\text {inter }}$ requires the availability of the Hilbert of $E^{\prime 2}$ inter to perform the phase shift associated with $\alpha_{3}$ and $\beta_{3}$ (see Section IV). One can verify that the following algebraic formula provides an exact evaluation of the Hilbert for multitone baseband excitations:

$$
\widehat{E^{\prime 2} \text { inter }}=\mathcal{H}\left(E^{\prime 2}{ }_{\text {inter }}\right)=2 I_{U}^{\prime} \widehat{I_{L}^{\prime}}+2 \widehat{I_{U}^{\prime}} I_{L}^{\prime}
$$

Note that the calculation of Hilbert transforms is costly and the proposed third-order algorithm presented above (and also its fifth-order extension) only requires the Hilbert transforms of $I$ and $Q$ since the remaining Hilbert transforms needed are all calculated from simple algebraic expressions.

To verify our VPD algorithm in the time domain, a Volterra model of RF PAs was implemented in MATLAB. The USB intermodulation of the RF PA model was phase shifted in the third Volterra Kernels to implement differential memory effects. The results are plotted in Figs. 10 and 11 for two- and tentone excitations, respectively. The results represented by the $\square$ symbol correspond to the case where interband corrections ( $H_{3 m}$ and $H_{3 p}$ ) are applied sequentially (serial connection) after the inband corrections $\left(H_{3 m m}, H_{3 m p}, H_{3 p m}, H_{3 p p}\right)$. The results 


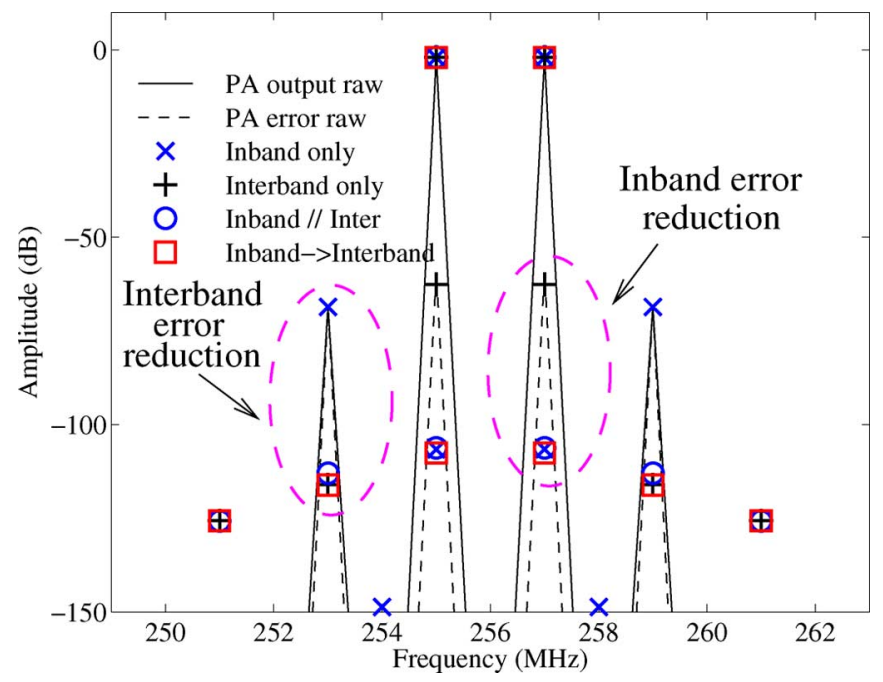

Fig. 10. Comparison of inband and interband VPD linearization in a RF PA with memory effects: two-tone case.

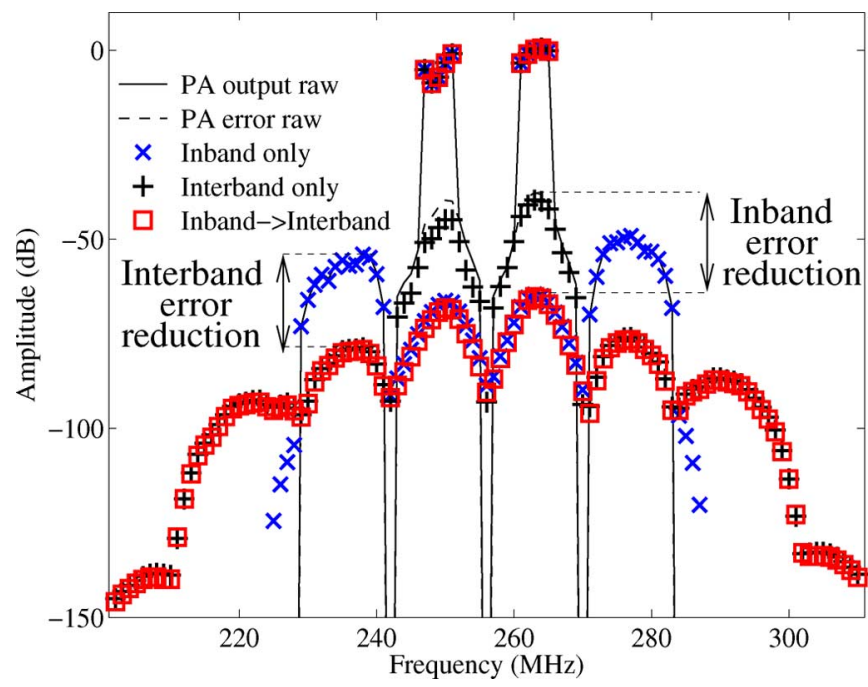

Fig. 11. Comparison of inband and interband VPD linearization in a RF PA with memory effects: ten-tone case.

represented by the o symbol correspond to the case where the inband and interband linearizations are performed in parallel. The results represented by the $x$ symbol correspond to inband linearization only and the + symbol to interband linearization only. As mentioned, the serial connection of the inband and interband linearization is slightly more effective in linearizing the Volterra model of an RF PA with differential memory effects. For the two-tone case, approximately $45-\mathrm{dB}$ reduction of both inband and interband distortion is obtained in Fig. 10. On the other hand, for the ten-tone case, shown in Fig. 11, the inband and interband intermodulations are reduced by approximately $25 \mathrm{~dB}$.

\section{FPGA IMPLEMENTATION OF FREQUENCY-SELECTIVE PREDISTORTION ALGORITHM}

The linearization algorithm was implemented with an FPGA. The FPGA test-bed used is shown in Fig. 12. The PA block is an $895-\mathrm{MHz}$ 10-W class-AB LDMOS RF ampliifer [2], [10]

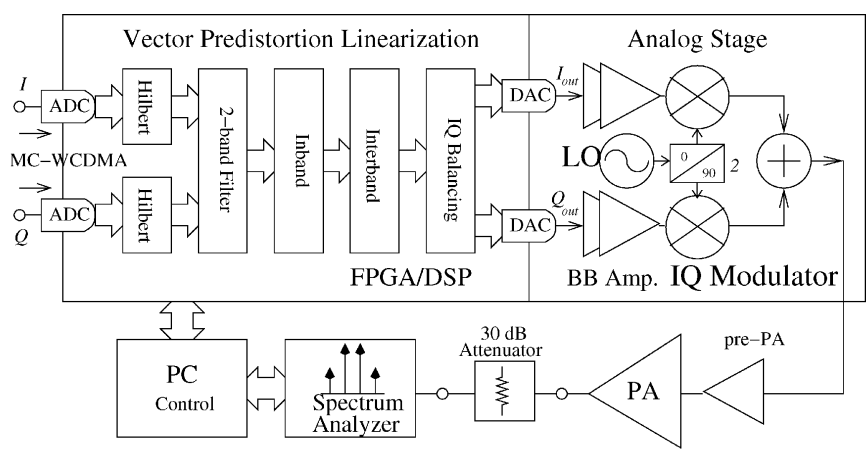

Fig. 12. Digital test-bed for baseband VPD.

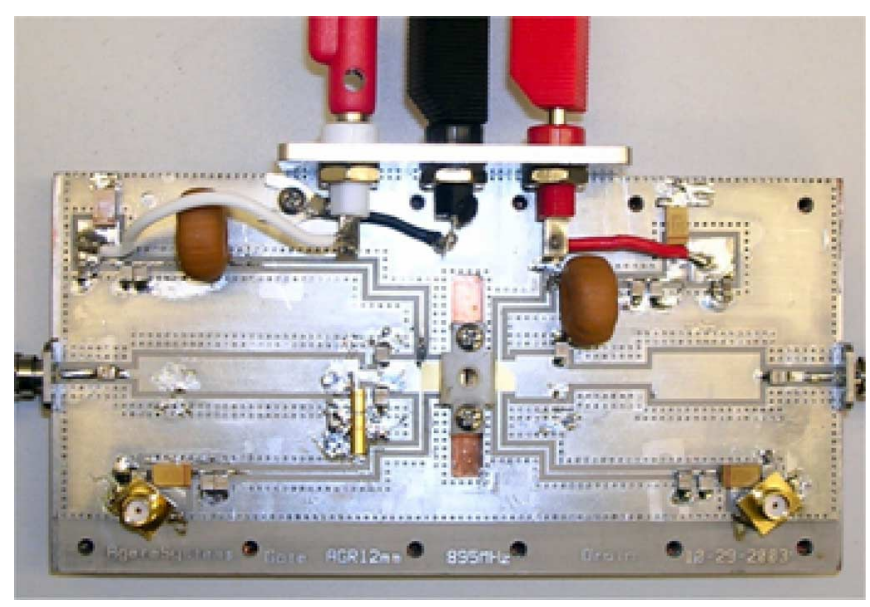

Fig. 13. 10-W class-AB RF PA operating at $895 \mathrm{MHz}$.

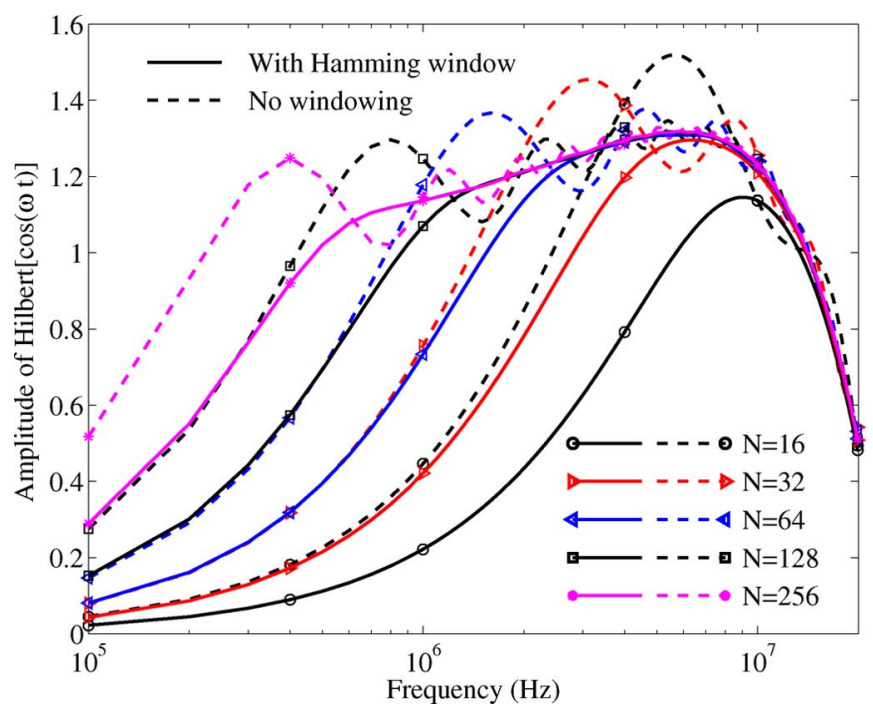

Fig. 14. Frequency-dependent amplitude of Hilbert transformation.

shown in Fig. 13. This digital test-bed is based on an Altera DSP board (EP1S25). This development board includes two in-board $\mathrm{A} / \mathrm{D}$ and $\mathrm{D} / \mathrm{A}$ converters. The two $\mathrm{A} / \mathrm{D}$ converters are 12 bits and the $2 \mathrm{D} / \mathrm{A}$ converters are 14 bits. The system clock $(100 \mathrm{MHz})$ is distributed via synchronized and delayed clocks to the A/D and D/A converters in the DSP board. Two-stage analog boards were developed for the adaptation of the signals between the DSP board and IQ modulator. The first stage increases the voltage 


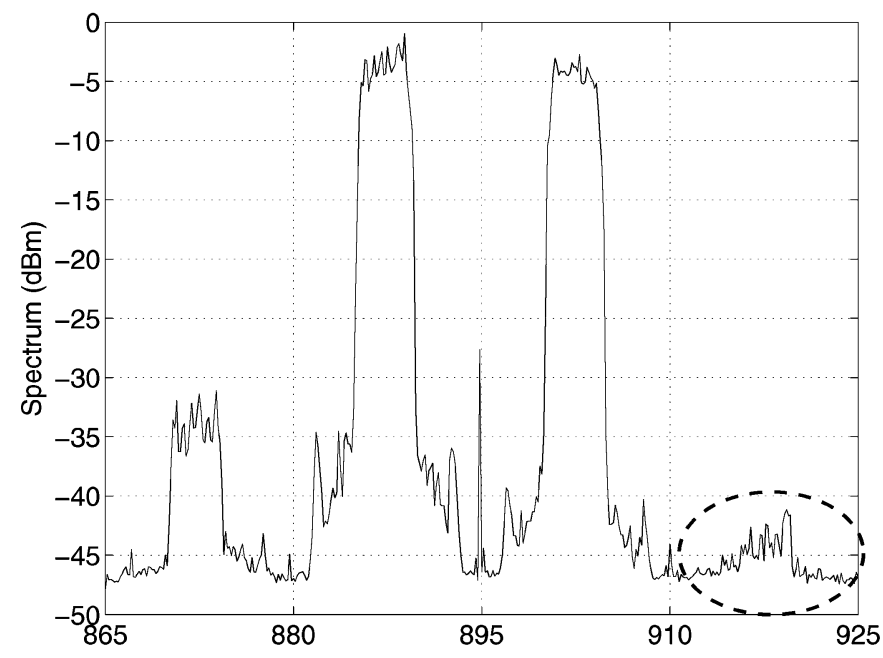

(a)

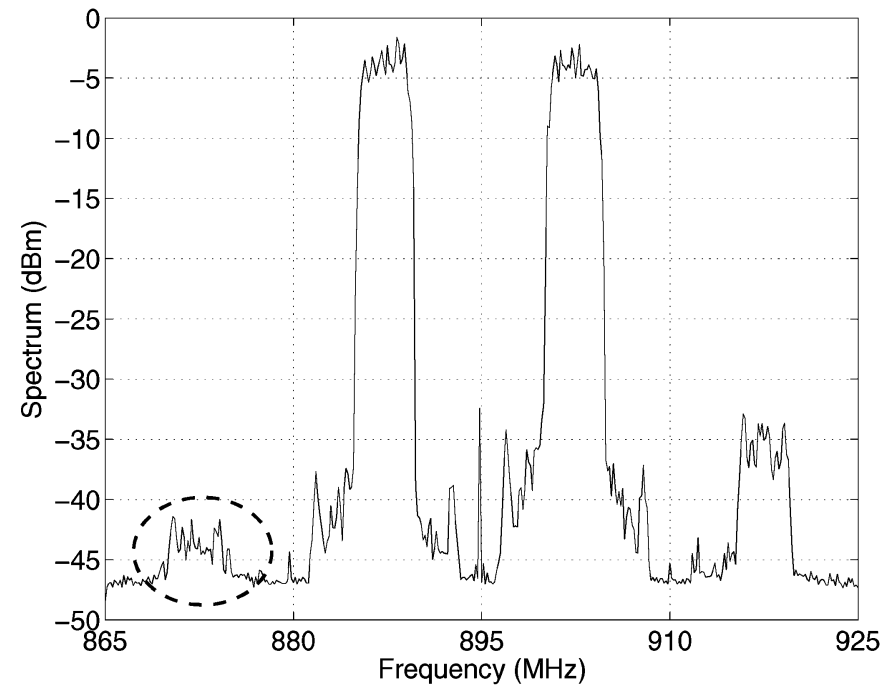

(c)

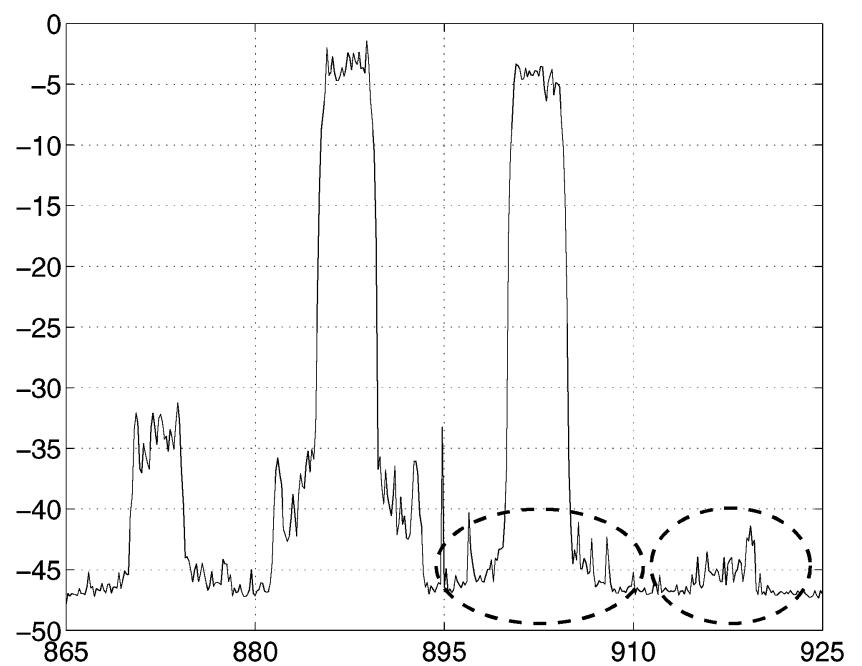

(b)

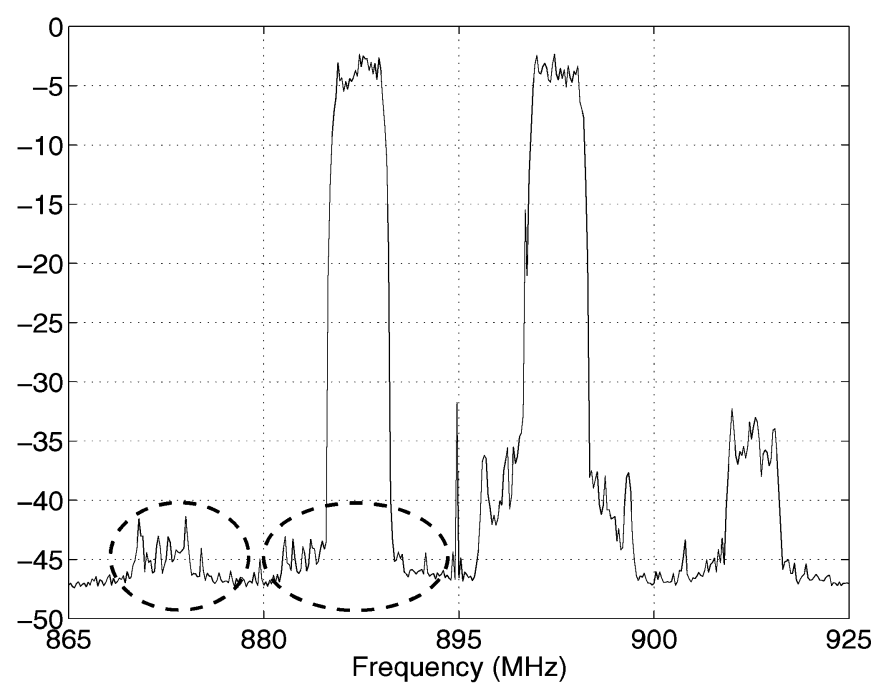

(d)

Fig. 15. Four-band frequency-selective: (a) USB interband, (b) USB inband and interband, (c) LSB interband, and (d) LSB inband and interband vectorial predistortion-linearization of a two-carrier WCDMA signal for a PA with differential memory.

level of the signals coming from the D/A converters and provides dc offset controls to manually adjust the LO leakage in the IQ modulator. The second stage analog board, which includes additional controls for the gain and differential dc offset, changes the mode of the IQ signals from single to complementary outputs to drive the differential IQ modulator.

In addition to the manual dc offset and gain controls implemented in the analog boards, several software blocks were introduced in the FPGA test-bed to control directly from Labwindows the dc offset (local oscillator (LO) leakage rejection) and the gain and phase correction required to compensate for the IQ imbalance of the IQ modulator. The methodology used for the IQ balancing has been reported in [15].

The two-band predistortion algorithm relies on two Hilbert transformations for the phase shifting of the I and Q signals and calculating the various envelopes and their Hilberts.

The digital implementation of the Hilbert transformation exhibits a number of limitation. This is revealed by inspecting the amplitude (Fig. 14) in the frequency domain of the Hilbert of a cosine input waveform for various conditions: number of taps (16-256) and Hamming window. While the phase (not shown) can be verified to be constant $\left(90^{\circ}\right)$ (no phase error), the amplitude is observed to vary as the modulation frequency is varied. The Hilbert algorithm used was implemented with 64 taps and a Hamming window to smooth the frequency response up to $10 \mathrm{MHz}$.

\section{PREDISTORTION LiNEARIZATION RESUlTS}

The performance of the proposed linearization algorithm was investigated using wideband signals (multitones and WCDMA signals) either generated using a vector source generator (ESG4438C) or directly synthesized from a lookup table (LUT) in the FPGA.

Frequency-selective balancing of the IQ modulator for the two inband band and the two intermodulation bands was performed before proceeding with the linearization. IQ balancing of 50 and $45 \mathrm{dBc}$ at the fundamental and third interband frequencies was achieved, respectively. 


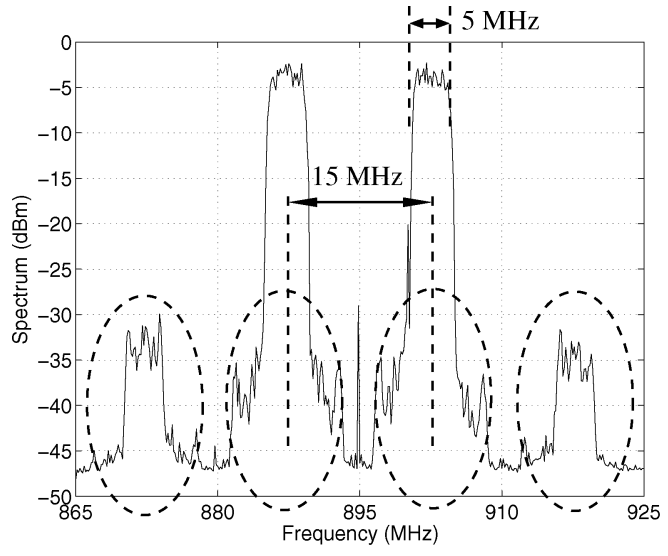

(a)

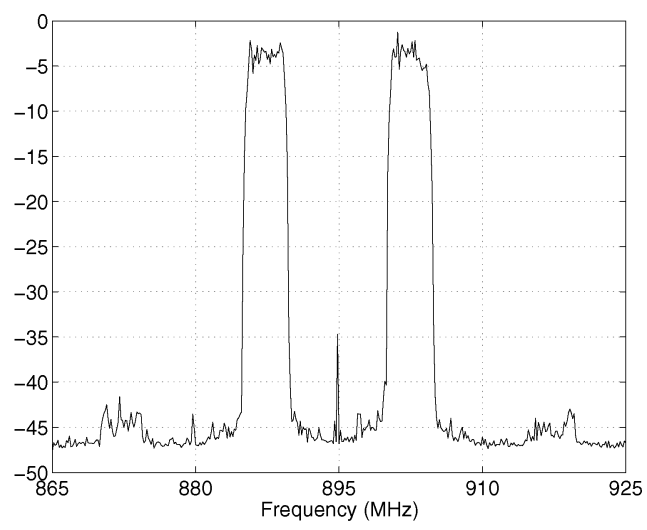

(b)

Fig. 16. (a) Nonlinearized spectrum and (b) joint inband and interband LSB and USB frequency-selective predistortion linearization of a two-carrier WCDMA signal in a PA with differential memory.

TABLE I

COMPARISON WITH OTHER PUBLISHED STUdies

\begin{tabular}{|c|c|c|c|c|}
\hline Source & Method & $\begin{array}{c}\text { ACPR } \\
(\mathrm{dBc})\end{array}$ & $\begin{array}{c}\Delta \text { ACPR } \\
(\mathrm{dB})\end{array}$ & $\begin{array}{c}\Delta f / f_{0} \\
(\mathbf{\%})\end{array}$ \\
\hline Kim [7] & D-BPD & -46 & $5-15$ & 0.77 \\
\hline Kubo [16] & D-BPD & -55 & $10-15$ & 0.70 \\
\hline Boumaiza [18] & D-RFPD & -40 & 12 & 0.76 \\
\hline Cha [17] & A-RFPD & -46 & 13.4 & 0.94 \\
\hline This work & D-BPD & -45 & 10 & 2.23 \\
\hline
\end{tabular}

Figs. 15 and 16 show the frequency-selective linearization of a two-carrier WCDMA signal for a 10-W LDMOS PA with 14-dB gain for $34.8-\mathrm{dBm}(3 \mathrm{~W})$ output power. The vertical scale is $5 \mathrm{~dB}$ per division and the horizontal scale is $15 \mathrm{MHz}$ per division for a total span of $60 \mathrm{MHz}$. Each WCDMA band has a 5-MHz bandwidth. The center of both bands is separated by $15 \mathrm{MHz}$. As indicated in Fig. 16(a), each band is immediately flanked on both sides by spectral regrowth bands of approximately $5-\mathrm{MHz}$ bandwidth, each originating from the interaction of each band with itself via $H_{3 m m}$ and $H_{3 p p}$ and the adjacent band via $H_{3 m p}$ and $H_{3 p m}$. In addition, both bands interfere together due to the terms $H_{3 m}$ and $H_{3 p}$ and generate two intermodulation bands at -22.5 and $+22.5 \mathrm{MHz}$ relative to the $\mathrm{LO}$ (center tone).

The ability of the frequency-selective predistortion linearization algorithm to reduce the spectral interband regrowth of either the LSB only or the USB only is demonstrated in Fig. 15(a) and (c). Further, as shown in Fig. 15(b) and (d), not only the intermodulation bands at -22.5 or $+22.5 \mathrm{MHz}$ can be reduced, but also the inband spectral regrowth surrounding the original band themselves. The proposed differential algorithm can separately address six types of inband and interband spectral regrowth in the LSB and USB.

Finally, Fig. 16(b) shows the combined reduction of the inband and interband spectral regrowth on both sides of the LO. These results demonstrate the capability of this algorithm to linearize a PA exhibiting differential memory effects. Note that the nonlinearities that extend over $50-\mathrm{MHz}$ bandwidth are reduced here by using real-time processing with no feedback loop required. The implementation of an adaptive algorithm for slow time variation should also be greatly facilitated by the frequency-selective nature of the linearization.

\section{CONCLUSION}

In this paper, third-order nonlinear system coefficients characterizing an LDMOSFET PA were extracted directly from LSNA measurements. These measurements revealed the presence of strong differential memory effects between the USB and LSB above $0.3 \mathrm{MHz}$. We proposed a novel predistortion algorithm, which accounts for asymmetry in nonlinearity between the LSB and USB. That algorithm relies on six parameters to independently address the third-order distortion while accounting for the differential memory effects of the RF PA. Independent control of the LSB and USB spectral regrowth was then demonstrated for two-carrier WCDMA signals for an overall $45 \mathrm{dBc}$ of ACPR. The extension of this theory to the linearization of fifth-order distortion has been demonstrated for multisine and will be reported elsewhere. As is shown in Table I, the worst case adjacent channel power ratio (ACPR) performance obtained compares well with other studies [7], [16]-[18], which reported experimental results for multicarrier PAs using the analog (A) or digital (D), baseband (B) or RF $(\mathrm{RF})$ predistortion $(\mathrm{PD})$ linearization methods. Also included for reference is the fractional bandwidth $\left(\Delta f / f_{0}\right)$. Note that the frequency-selective algorithm is demonstrated here with more than twice the fractional bandwidth of these previous studies. Finally note that the present frequency-selective topology could be combined with other linearization techniques (e.g., memory polynomials [7], [8] ) for further improved performance.

The extension from two-carrier to multicarrier PAs can proceed by further dividing the bandwidth in additional bands [19] and applying the present algorithm to each individual pair of bands. An adaptive algorithm then becomes desirable to automatically calibrate the linearization [9], [20]. The implementation of such a calibration will be facilitated by the fact that each complex linearization coefficient can be extracted independently as verified in this study for two bands. 


\section{ACKNOWLEDGMENT}

This paper is dedicated to the memory of late Prof. K. Breeding, The Ohio State University, Columbus, whose development of the OSU Digital Educational Laboratory made this study possible. The authors are also indebted to the Altera Corporation, San Jose, CA, for the donation of the FPGA DSP test-bed used in this study. Finally, the authors are grateful to the reviewers for their comments on this paper's manuscript, which greatly improved its clarity.

\section{REFERENCES}

[1] J. S. Kenney, W. Woo, L. Ding, R. Raich, H. Ku, and G. T. Zhou, "The impact of memory effects on predistortion linearization of RF power amplifiers," in Proc. 8th Int. Microw. Opt. Tech. Symp., Montreal, QC, Canada, Jun. 19-23, 2001, pp. 189-93.

[2] W. Dai and P. Roblin, "Distributed and multi-time-constant electrothermal modeling and its impact on ACPR in RF predistortion," in 62th ARFTG Conf. Dig., Denver, CO, Dec. 2003, pp. 89-98.

[3] J. Vuolevi and T. Rahkonen, Distortion in RF Power Amplifiers. Norwood, MA: Artech House, 2003.

[4] R. Meyer, R. Eschenback, and W. Edgerley, Jr., "A wideband feedforward amplifier," IEEE J. Solid-State Circuits, vol. SCC-9, no. 6, pp. 422-448, Jun. 1974.

[5] W. Woo, M. D. Miller, and J. S. Kenney, "A hybrid digital/RF envelope predistortion linearization system for power amplifiers," IEEE Trans. Microw. Theory Tech., vol. 53, no. 1, pp. 229-237, Jan. 2005.

[6] J. Pedro and S. A. Maas, "A comparative overview of microwave and wireless power-amplifier behavioral modeling approaches," IEEE Trans. Microw. Theory Tech., vol. 53, no. 4, pp. 1150-1163, Apr. 2005.

[7] J. Kim and K. Konstantinou, "Digital predistortion of wideband signals based on power amplifier model with memory," Electron. Lett., vol. 37, no. 23, pp. 1417-1418, Dec. 2001.

[8] L. Ding, G. T. Zhou, D. R. Morgan, Z. Ma, J. S. Kenney, J. Kim, and C. R. Giardina, "A robust digital baseband predistorter constructed using memory polynomials," IEEE Trans. Commun., vol. 52, no. 1, pp. 159-165, Jan. 2004

[9] R. Sperlich, J. A. Sills, and J. S. Kenney, "Closed-loop pigtail predistortion with memory effects using digital pre-distortion and genetic algorithms," in IEEE MTT-S Int. Microw. Symp. Dig., Jun. 2005, pp. $1557-1560$.

[10] S. K. Myoung, D. Chaillot, P. Roblin, W. Dai, and S. J. Doo, "Volterra characterization and predistortion linearization of multi-carrier power amplifiers," in 64th ARFTG Conf. Dig., Orlando, FL, Dec. 2004, pp. 65-73.

[11] A. A. M. Saleh, "Matrix analysis of mildly nonlinear, multiple-input, multiple-output systems with memory," Bell Syst. Tech. J., vol. 61, no. 9, pp. 2221-2243, Nov. 1982.

[12] M. Schetzen, "Nonlinear system modeling based on the Wiener theory," Proc. IEEE, vol. 69, no. 12, pp. 1557-1573, Dec. 1981.

[13] J. J. Bussgang, L. Ehrman, and J. W. Graham, "Analysis of nonlinear systems with multiple inputs," Proc. IEEE, vol. 62, no. 12, pp. 1088-1119, Dec. 1974.

[14] J. Verspecht, F. Verspecht, and M. Vanden Bossche, "Network analysis beyond $S$-parameters," in 56th ARFTG Conf. Dig., Broomfield, CO, Dec. 2000, pp. 1-4.

[15] S. K. Myoung, X. Cui, P. Roblin, D. Chaillot, F. Verbeyst, M. Vanden Bossche, S. J. Doo, and W. Dai, "Large signal network analyzer with trigger for baseband modulation linearization," in 64th ARFTG Conf. Dig., Orland, FL, Dec. 2004, pp. 189-195.

[16] T. Kubo, N. Fudaba, H. Ishikawa, H. Hamada, K. Nagatani, H. Hayashi, T. Maniwa, and Y. Oishi, "A highly efficient adaptive digital predistortion amplifier for IMT-2000 base stations," in Veh. Technol. Conf., 2003, vol. 4, pp. 2206-2210.

[17] J. Cha, J. Yi, J. Kim, and B. Kim, "Optimum design of a predistortion RF power amplifier for multicarrier WCDMA applications," IEEE Trans. Microw. Theory Tech., vol. 52, no. 2, pp. 655-663, Feb. 2004.

[18] S. Boumaiza, J. Li, M. Jaidane-Saidane, and F. Ghannouchi, "Adaptive digital/RF predistortion using a nonuniform LUT indexing function with built-in dependence on the amplifier nonlinearity," IEEE Trans. Microw. Theory Tech., vol. 52, no. 12, pp. 2670-2677, Dec. 2004.
[19] O. Hammi, S. Boumaiza, M. Jaïdane-Saïdane, and F. M. Ghannouchi, "Digital subband filtering predistorter architecture for wireless transmitters," IEEE Trans. Microw. Theory Tech., vol. 53, no. 5, pp. 1643-1652, May 2005.

[20] N. Safari, J. P. Tanem, and T. Roste, "A block-based predistortion for high-power amplifier linearization," IEEE Trans. Microw. Theory Tech., vol. 54, no. 6, pp. 2813-2820, Jun. 2006.

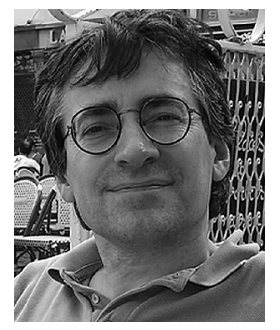

Patrick Roblin (M'85) was born in Paris, France, in September 1958. He received the Maitrise de Physics degree from the Louis Pasteur University, Strasbourg, France, in 1980, and the M.S. and D.Sc. degrees in electrical engineering from Washington University, St. Louis, MO, in 1982 and 1984, respectively.

In 1984, he joined the Department of Electrical and Computer Engineering, The Ohio State University (OSU), Columbus, where he is currently a Professor. He is the founder of the Non-Linear RF Research Laboratory, OSU. He developed two educational $\mathrm{RF} /$ microwave laboratories and associated curriculum for training senior undergraduate and graduate students at OSU. He coauthored the textbook High-Speed Heterostructure Devices (Cambridge Univ. Press, 2002). His current research interests include the measurement, modeling, design, and linearization of nonlinear RF devices and circuits such as oscillators, mixers, and PAs.

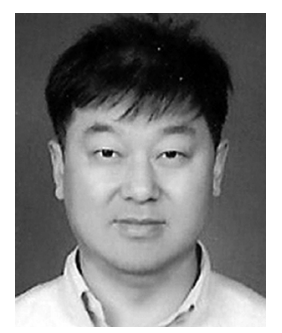

Suk Keun Myoung was born in Anyang, Kyunggi-do, Korea, in September 1968. He received the B.S. degree in mathematical education and M.S. degree in physics from Korea University, Seoul, Korea, in 1994 and 1996, respectively, and the $\mathrm{Ph} . \mathrm{D}$. degree in electrical and computer engineering from The Ohio State University, Columbus, in 2006.

From 1996 to 2000, he has was a Researcher with Korea Telecom. He is currently with the System Core Technology Laboratory, Telecommunication Research and Development Center, Samsung, Suwon, Gyeonggi, Korea. His current research concerns adaptive digital predistortion for WiMax basestations.

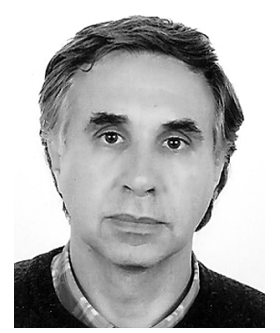

Dominique Chaillot was born in Brive, France, in October 1963. He received the Maitrise de Physics degree from the Universite des sciences, Laboratoire IRCOM (now XLIM), Limoges, France, in 1985, the $\mathrm{Ph} . \mathrm{D}$. degree in electrical engineering from the IRCOM Laboratory, Limoges University, Limoges, France, in 1989, and the Master of Business Administration degree from Sorbonne University, Paris, France, in 1992.

In 1990, he joined the Commissariat à l'énergie atomique (CEA) (the French Atomic Agency), Gif sur Yvette, France, as a Research Engineer. He is currently on leave with the Department of Electrical and Computer Engineering, The Ohio State University (OSU), Columbus, where he is an Invited Scholar. His expertise is on real-time signal-processing in RF systems. His current research interests include the measurement, design, and linearization of nonlinear RF devices and PAs.

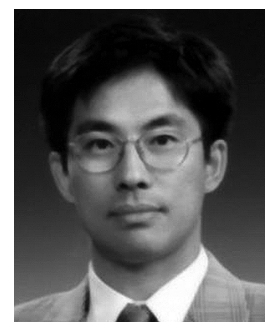

Young Gi Kim (M'00) was born in Seoul, Korea. He received the B.S. and M.S. degrees in electronics engineering from Hanyang University, Seoul, Korea, in 1983 and 1984, respectively, and the Ph.D. degree from the University of Texas at Arlington, in 1993.

From 1986 to 1997, he was with the Korea Telecom Research Laboratory, where he was engaged with long-distance optical fiber communication and developed monolithic microwave integrated circuits for wireless application. In 1996 he joined the Department of Data Communication Engineering, Anyang University, Gyeonggi-do, Korea, where he is currently a Professor. From 2005 to 2006, he was a Visiting Professor with the Mixed Signal Electronics Laboratory, The Ohio State University (OSU). His research interests include the design of RF integrated circuits and devices. 


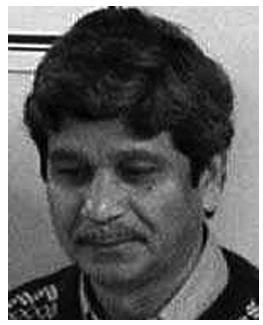

Ayub Fathimulla received the B.Sc. degree in physics from Sri Venkateswara (S.V.) University, Tirupati, India, in 1963, the B.E. degree in electronics and communications engineering from Andhra University, , InVisakhapatnamdia, in 1966, the M.E. degree in electronics and communiation engineering from Osmania University, Hyderabad, India, in 1968, and the Ph.D. degree in electrical engineering from Rensselaer Polytechnic Institute (RPI), Troy, NY, in 1980.

He is currently Vice President of Technology and Product Development with Epitaxial Technologies LLC, Baltimore, MD. He has developed and led the development of various optical, ultrahigh-speed digital, and microwave compound semiconductor technologies with Allied Signal (Honeywell). He was most recently Chief Engineering Fellow with Honeywell during which time he was responsible for developing and transferring microwave silicon-on-insulator (SOI)-CMOS technology to production.

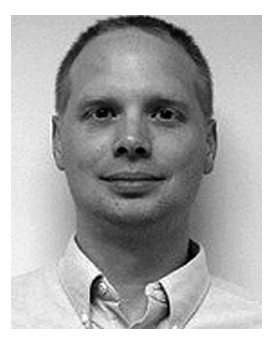

Jeff Strahler received the B.S.E.E. degree from the University of Cincinnati, Cincinnati, OH, in 1989, and the M.S.E.E. degree in electrical engineering from The Ohio State University (OSU), Columbus, in 1991.

He completed his master's thesis in the area of computational electromagnetics with the ElectroScience Laboratory, Department of Electrical and Computer Engineering, OSU. From 1986 to 1990, he was involved in different roles for Comsat Laboratories, during which time he designed microwave circuits and antennas for communication satellite and earth station systems. In 1991, he joined AT\&T Bell Laboratories (now Alcatel-Lucent), Columbus,
$\mathrm{OH}$, as a Member of the Technical Staff (MTS) and later as a Distinguished Member of the Technical Staff (DMTS). As part of his duties, he has been a Team Leader for the design and development of wireless basestation amplifiers for AMPS, TDMA, GSM, and CDMA systems. In June 2001 he joined the Celiant Corporation, which was acquired by the Andrew Corporation, Westchester, IL, in June 2002. He was recently appointed an Andrew Fellow, where he continues research and develop activities for basestation PA products.

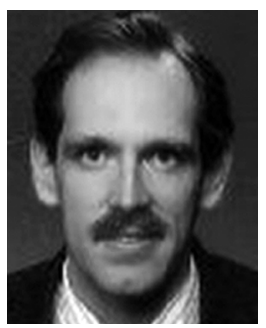

Steven Bibyk (S'78-M'80) received the combined B.S./M.S. and Ph.D. degrees from Case Western Reserve University, Cleveland, OH, in 1980 and 1983, respectively, all in electrical engineering and applied physics.

In 1984, he joined The Ohio State University, Columbus, where he teaches courses in circuit design, communication systems, and project design. He has been a Visiting Research Scientist with the Space Electronics Division, National Aeronautics and Space Administration (NASA) Glenn Research Center and the Ohio Aerospace Institute, and was a Member of the Technical Staff with Sandia National Laboratories, Albuquerque, NM. He has authored or coauthored over 60 technical papers. He has advised 13 Ph.D. students and approximately 40 M.S. students to completion. His research concerns information systems in very large scale integration (VLSI), system-on-chip design methods, and RF/mixed-signal circuit design.

Dr. Bibyk is a Life Member of the American Physical Society. He is a member of Tau Beta Pi and Eta Kappa Nu. He was a two-time recipient of a NASA Summer Faculty Fellowship and a National Research Council (NRC) fellowship with the U.S. Air Force. 